\title{
Immunochemical Analysis of Relationships Among Isolated Frankiae (Actinomycetales)
}

\author{
DWIGHT BAKER,† WILLIAM L. PENGELLY, $\ddagger$ AND JOHN G. TORREY \\ Cabot Foundation for Botanical Research, Harvard University, Harvard Forest, \\ Petersham, Massachusetts 01366
}

\begin{abstract}
An immunological study of nine strains of pure-cultured members of the order Actinomycetales isolated from symbiotic root nodules was undertaken to establish the taxonomic relationships of these organisms. Based on the data from a simple double-diffusion (Ouchterlony) precipitation procedure, these isolates, which tentatively have been classified in the genus Frankia (family Frankiaceae), could be divided into two major groups. The first group, designated serotype I, included all of the Actinomycetales strains isolated from Alnus, Comptonia, and Myrica host plants. The second group, serotype II, included Actinomycetales strains isolated from Elaeagnus host plants. No ambiguous cross-reactions with unrelated members of the Actinomycetales were observed. Crushed nodule suspensions cross-reacted in the same manner as pure-cultured frankiae, suggesting that the technique could be important in screening for novel, naturally occurring strains. A comparative study in which an immunofluorescence procedure was used failed to show reliable correlations among the Actinomycetales strains tested. We concluded that the immunodiffusion technique was the better of the two methods for Frankia strain characterization.
\end{abstract}

A microsymbiont from a nitrogen-fixing actinorhizal plant symbiosis was first isolated and cultured in vitro by Callaham et al. (7). Since then, additional actinorhizal microsymbionts have been successfully isolated and cultured in vitro (2). All of these filamentous bacteria have been assigned tentatively to the genus Frankia of the family Frankiaceae (10). Scientific names have not been applied to these isolates since there are discrepancies in the current taxonomic classification based on host ranges, as proposed by Becking (5). In addition to information on host range, chemotaxonomic characters (10) and cultural and morphological characters $(1,3)$ have been offered as aids in distinguishing individual microorganisms. However, these characters alone cannot be used as ultimate deciding factors since Frankia strains isolated from very different hosts are often indistinguishable in the laboratory (6).

Lalonde et al. (9) used an immunolabeling procedure to show the affinity of a noninfective Frankia-like organism to nodule endophytes. In this study, antibodies were formed against nodule isolates or a "purified endophyte suspension," conjugated with fluorescein isothiocyanate, and used as a reagent stain against the cultured organism or other endophyte suspensions. From their tests, these workers attempted

+ Present address: Department of Biology, Middlebury College, Middlebury, VT 05753.

* Present address: Department of Botany and Plant $\mathrm{Pa}$ thology, Michigan State University, East Lansing, MI 48824. to show that their in vitro-grown isolate was identical to the in vivo nodule organism. However, results were ambiguous, because the immunoglobulin $\mathrm{G}$ fractions which were used crossreacted with two significantly different cultured bacterial strains. Recently, it has been determined (2) that in fact the organism which Lalonde et al. isolated in vitro was not a Frankia species, thus further confusing the data of these workers.

In an effort to determine whether immunochemical methods might provide a simple and relatively rapid in vitro test for distinguishing strains of Frankia, we compared an immunofluorescence procedure and a double-diffusion (Ouchterlony) precipitin reaction technique. The confirmed Frankia strains CpI1 and EuI1 were used as immunoantigens.

\section{MATERIALS AND METHODS}

Bacterial strains. The strains used in this study are listed in Table 1.

Preparation of antisera. Cultures of Frankia strains CpI1 (7) and EuI1 (4) were grown in a yeast extract-glucose medium (Frankia broth) (2) for 6 weeks at $28^{\circ} \mathrm{C}$. The mycelia were harvested by centrifugation, washed twice in sterile distilled water, and homogenized by ultrasonic disruption with a Braunsonic model 1510 Ultrasonifier $(50 \mathrm{~W}$ for two $15-\mathrm{s}$ intervals). These cell suspensions were then freezedried, and the dry residue was used as a stock for all injection procedures. The cells were neither killed nor fixed.

Two rabbits were used for antibody preparation for 
TABLE 1. Strains of Actinomycetales used in this study

\begin{tabular}{lcll}
\hline \multicolumn{1}{c}{ Taxon } & Strain designation & \multicolumn{1}{c}{ Source plant } & Source $^{a}$ \\
\hline D. congolensis & $130 / 75$ & & Lechevalier \\
Frankia sp. & AvcI1 & Alnus viridis subsp. crispa & Baker \\
Frankia sp. & AvsI2 & A. viridis subsp. sinuata & Baker \\
Frankia sp. & ArI3 & Alnus rubra & Berry \\
Frankia sp. & ArI4 & A. rubra & Baker \\
Frankia sp. & CpI1 & Comptonia peregrina & Callaham \\
Frankia sp. & CpI2a & C. peregrina & Baker \\
Frankia sp. & EuI1 & Elaeagnus umbellata & Baker \\
Frankia sp. & EuI5 & E. umbellata & Baker \\
Frankia sp. & MpI1 & Myrica pensylvanica & Lechevalier \\
Streptomyces albus & IMRU 758 & & Lechevalier \\
S. roseum & $1-18$ & & Lechevalier \\
\hline
\end{tabular}

${ }^{a}$ Baker, Strains isolated by D.B. by a sucrose density isolation procedure; Berry, A. Berry, Cabot Foundation for Botanical Research, Harvard University, Petersham, Mass.; Callaham, D. Callaham, Cabot Foundation for Botanical Research, Harvard University; Lechevalier, M. P. Lechevalier, Waksman Institute of Microbiology, Rutgers University, Piscataway, N.J.

$b$ This source plant had been inoculated and nodulated by Frankia strain CpI1.

c The same source plant was used for the isolations of Frankia strains EuI1 and EuI5.

each of the Frankia strains. Before an injection series was undertaken, a small amount of blood was collected from each of the rabbits for comparative purposes; this sample is referred to as the control serum. For each bacterial strain, one rabbit was immunized intravenously by injecting approximately $500 \mu \mathrm{g}$ (dry weight) of antigen twice each week over a period of 4 weeks. Booster immunizations were administered after a resting interval of $\mathbf{4}$ months by injecting an equivalent amount of antigen over a period of 2 weeks. Blood samples were collected on day 3 after the last injection of each series. The second rabbit for each strain was immunized subcutaneously. Two injections of 5 to 7 mg (dry weight) of antigen were given 2 weeks apart. Similar booster injections were administered after resting periods of $\mathbf{4}$ months. Incomplete Freund adjuvant was added to the antigen for the first injection of each series. Blood samples were collected 7 to 10 days after the second injection of each series.

For both injection procedures, the freeze-dried antigen was rehydrated in approximately $1 \mathrm{ml}$ of $0.15 \mathrm{M}$ $\mathrm{NaCl}$ and homogenized in a Potter-Elvehjem tissue grinder to prevent sedimentation of the cells during injection. Blood samples collected from the marginal ear vein were permitted to clot at room temperature and then refrigerated overnight. Serum was decanted, cleared of cells by centrifugation, made $0.1 \%$ (wt/vol) in sodium azide, sterilized by membrane filtration (0.45- $\mu \mathrm{m}$ filters; Millipore Corp., Bedford, Mass.), and stored at $4^{\circ} \mathrm{C}$. The sera used in the procedures described below were from the blood samples collected after the first or second booster injection series.

Preparation of cells for immunochemical tests. Each of the bacterial strains was cultured in Frankia broth; the cells were harvested by centrifugation, washed twice in sterile physiological saline, and then homogenized by sonication as described above. For the immunodiffusion assay, crushed nodule suspensions were prepared by grinding root nodules in a mortar and pestle with physiological saline. This suspension was then filtered through Miracloth (Chicopee Mills, Milltown, N.J.) and glass wool and then sonicated as described above.
Immunofluorescence assays. Mycelial suspensions of the strains were tested for cross-reactions with whole sera from intravenously injected rabbits by using an indirect immunofluorescence technique (8). Small amounts of each bacterial suspension (volume, 10 to $25 \mu$ l of packed cells) were added to disposable glass culture tubes ( 6 by $50 \mathrm{~mm}$; Fisher Scientific Co., Pittsburgh, $\mathrm{Pa}$.) and mixed with antiserum to give a final antibody titer ranging from 1:5 to 1:200 with phosphate-buffered saline $\left(0.01 \mathrm{M} \mathrm{KH}_{2} \mathrm{PO}_{4}, 0.15 \mathrm{M}\right.$ $\mathrm{NaCl}, \mathrm{pH} 7.4$ ) in a final volume of $0.2 \mathrm{ml}$. Mixtures were incubated for $15 \mathrm{~min}$ and centrifuged, and the bacterial pellets were washed three times with $0.2 \mathrm{ml}$ of phosphate-buffered saline. The bacteria were then suspended in $0.2 \mathrm{ml}$ of phosphate-buffered saline containing goat anti-rabbit immunoglobulin $\mathrm{G}$ conjugated with fluorescein isothiocyanate (ICN Nutritional Biochemicals, Cleveland, Ohio). Goat antibody titers ranged from 1:5 to $1: 100$. The mixtures were incubated for $15 \mathrm{~min}$ at room temperature and centrifuged, and the bacteria were washed three times with $0.2 \mathrm{ml}$ of phosphate-buffered saline. Observation of the organisms after treatment with antisera was performed with a Leitz-Wetzlar Ortholux microscope equipped with an epi-illumination ultraviolet light source.

Ouchterlony immunodiffusion assays. The study strains were tested for cross-reactions with whole sera from subcutaneously injected rabbits by using a double-diffusion technique (11). Diffusion plates $(60 \mathrm{~mm})$ containing a $0.1 \% \mathrm{NaN}_{3}$-preserved 0.15 $\mathrm{M} \mathrm{NaCl}$ solution solidified with $1.5 \%$ Noble agar (Difco) were prepared. Wells were formed by pouring the agar solution around Penicylinders (Fisher Scientific) arranged in a template pattern. Mycelial suspensions were loaded into the outer wells. Whole serum samples were loaded into the center wells. Each well was filled with $250 \mu \mathrm{l}$ of material. The diffusion plates were incubated in a moist chamber at $4^{\circ} \mathrm{C}$ for 10 days.

\section{RESULTS}

Immunofluorescence assays. Immunofluorescence labeling studies showed no strong spec- 
ificity in antiserum preparations for the strains used as inocula. All of the strains tested reacted with the two antisera to some degree, and no reaction was observed between the study strains and the control serum (Table 2). Attempts to show specificity of the immunoreaction by diluting antisera or fluorescent antibody preparations failed, and antibody dilution reduced the intensity of labeling in all cases. Thus, the intensities of reactions with different strains overlapped to the extent that results could not be evaluated semi-quantitatively. Slight autofluorescence by some strains further confused the data. Because of the extensive cross-reactivity found, no attempt was made to improve specificity by absorbing antisera with heterologous bacteria. Instead, the strains were analyzed by immunodiffusion.

Immunodiffusion assays. Representative immunodiffusion plates are shown in Fig. 1 through 6. No precipitin lines were observed when the strains were challenged with the control sera (Fig. 1). The antiserum detected a common class or group antigen among all of the Alnus, Comptonia, and Myrica isolates (Fig. 2, 4, and 5, large arrows) but not in a strain of Streptosporangium roseum (Fig. 2) or a strain of Dermatophilus congolensis (Fig. 4). Homologous reactions were observed between antiserum to CpI1 and Frankia strains AvcI1, AvsI2, ArI3, ArI4, CpI1, CpI2a, and MpI1 (Fig. 2 and 4 through 6). Heterologous reactions were observed between antiserum to $\mathrm{CpI} 1$ and Frankia strains EuI1 (Fig. 2) and EuI5. Similarly, homologous reactions were observed between antiserum to EuI1 and strains EuI1 and EuI5 (Fig. $3)$. In most cases, homologous reactions were indicated by one intense major precipitin band and one or two minor bands. Occasionally, additional minor precipitin bands were observed

TABLE 2. Immunofluorescent reactions of purecultured organisms to anti-Frankia sera

\begin{tabular}{lrcc}
\hline & \multicolumn{3}{c}{$\begin{array}{c}\text { Immunofluorescent reac- } \\
\text { tion with: }\end{array}$} \\
\cline { 3 - 4 } Antigen & $\begin{array}{c}\text { Strain designa- } \\
\text { tion }\end{array}$ & $\begin{array}{c}\text { Anti-CpI1 } \\
\text { serum }\end{array}$ & $\begin{array}{c}\text { Anti-EuIl } \\
\text { serum }\end{array}$ \\
\hline D. congolen- & $130 / 75$ & $+^{a}$ & $+^{a}$ \\
$\quad$ sis & & & \\
Frankia sp. & AvcI1 & + & + \\
Frankia sp. & ArI3 & + & + \\
Frankia sp. & CpI1 & + & + \\
Frankia sp. & CpI2a & + & + \\
Frankia sp. & EuI1 & + & + \\
Frankia sp. & MpI1 & + & + \\
Streptomyces & IMRU 758 & + & + \\
albus & & & + \\
\hline
\end{tabular}

${ }^{a}$ This organism was autofluorescent in the absence of antiserum.
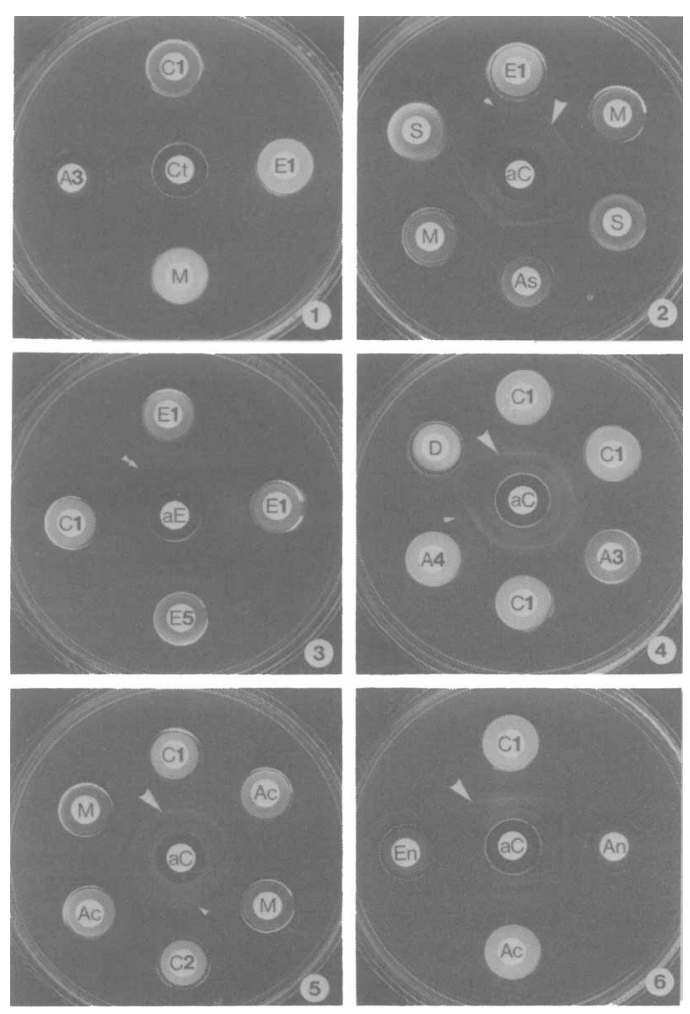

FIG. 1. Immunodiffusion analysis of actinorhizal microsymbiont strains. Abbreviations: Ct, control rabbit serum; A3, ArI3; C1, CpI1; E1, EuI1; $M, M p I 1$; Note the absence of any precipitin reaction between the rabbit control serum and the four isolated Frankia strains.

FIG. 2. Cross-reactions of antiserum to strain CpII (aC) with various organisms. Note the intense homologous precipitin band (large arrow) with the closely related Frankia strains $M p I 1(M)$ and AvsI2 (As), the nonhomologous precipitin band (small arrow) with strain EUI1 (E1), and the lack of reaction with the $S$. roseum (S) strain (Actinoplanaceae).

FIG. 3. Cross-reaction of antiserum to strain EuI1 (aE) with Frankia strains. Note the homologous precipitin band (double arrow) with the closely related Elaeagnus-isolated strains EuI1 (E1) and EuI5 (E5) and the lack of reaction with strain CpI1 (C1).

FIG. 4. Cross-reactions of antiserum to CpII (aC) with various organisms. Note the intense homologous precipitin band (large arrow) with the closely related Frankia strains and the lack of reaction with the $D$. congolensis (D) strain (Dermatophilaceae); the less intense secondary band (small arrow) may suggest subtle differences among the isolates. Abbreviations: C1, CpI1; A3, ArI3; A4, ArI4.

Fig. 5. Cross-reactions of antiserum to strain $\mathrm{CpI}$ $(a C)$ with isolated Frankia strains. Note the intense homologous precipitin band (large arrow) with all closely related strains; again, a secondary band (small arrow) suggests subtle differences among the strains. Abbreviations: C1, CpI1;Ac, AvcI1; M, MpI1; C2, $C p I 2 a$. 
(Fig. 4 and 5), thus suggesting subtle differences between the isolated frankiae, differences which may be useful in future taxonomic studies. Crushed nodule suspensions prepared from nodules initiated by pure cultures cross-reacted in the same manner as the original microorganism (Fig. 6); however, the in vitro-cultivated strain showed the presence of additional individual antigens.

\section{DISCUSSION}

The results reported here show that a simple immunodiffusion technique is capable of distinguishing among the frankiae and other purecultured organisms. The close homology of several Frankia strains suggests that differences between strains are often subtle, a conclusion supported by our inability to detect differences by immunofluorescence methods. Therefore, immunodiffusion methods, which can readily detect qualitative differences in bacterial antigens, are more useful than fluorescence labeling methods for characterizing strain differences within the Frankiaceae. Further refinement of the diffusion method, such as the use of immunoelectrophoresis, may be helpful in defining differences between closely related strains.

The results of this study suggest that among the isolated frankiae tested, two taxonomic groups may be described. The first group consists of organisms which were isolated from root nodules of Alnus, Comptonia, and Myrica and which had a common serological marker. We designate this group serotype I. The second group consists of organisms isolated from root nodules of Elaeagnus; these organisms also had a common serological marker, but one that was different from that of serotype I. We designate this group serotype II.

Results from physiological and chemical studies, as well as cross-inoculation tests of some of the isolated frankiae, support the hypothesis that there are two distinct taxonomic groups, as described above $(1,2,10)$. Additional taxonomic groups will no doubt be distinguished as novel organisms are isolated from other actinorhizal genera.

One additional asset of the immunodiffusion technique is the ability to distinguish serologi-

Fig. 6. Cross-reactions of antiserum to $\mathrm{CpI1}(\mathrm{aC})$ with isolated Frankia strains or nodule suspensions. Note the homologous precipitin band (large arrow) with the closely related strains $\mathrm{CpII}$ (C1) and AvcII (Ac) and with the suspension of nodules induced by AvcI1 (An) and the lack of a precipitin band with the nodule suspension induced by EuII (En). cally distinct strains from crushed root nodule suspensions in the absence of isolated pure cultures. This may prove to be useful in the selection of naturally occurring strains for isolation attempts.

\section{ACKNOWLEDGMENTS}

We thank $\mathrm{W}$. Ormerod for photographic assistance and Alison Berry, Dale Callaham, and Mary P. Lechevalier for supplying bacterial strains.

This research was supported in part by the Maria Moors Cabot Foundation for Botanical Research, Harvard University, and by grant DEB-77-02249 from the National Science Foundation.

\section{REPRINT REQUEST'S}

Address reprint requests to: Dwight D. Baker, Department of Biology, Middlebury College, Middlebury, VT 05753.

\section{LITERATURE CITED}

1. Baker, D., W. Newcomb, and J. G. Torrey. 1980. Characterization of an ineffective actinorhizal microsymbiont, Frankia sp. EuI1 (Actinomycetales). Can. J. Microbiol. 26:1072-1089.

2. Baker, D., and J. G. Torrey. 1979. The isolation and cultivation of actinomycetous root nodule endophytes, p. 38-56. In J. C. Gordon, C. T. Wheeler, and D. A. Perry (ed.), Symbiotic nitrogen fixation in the management of temperate forests. Forestry Research Laboratory, Oregon State University, Corvallis.

3. Baker, D., and J. G. Torrey. 1980. Characterization of an effective actinorhizal microsymbiont, Frankia sp. AvcIl (Actinomycetales). Can. J. Microbiol. 26:10661071.

4. Baker, D., J. G. Torrey, and G. H. Kidd. 1979. Isolation by sucrose-density fractionation and cultivation in vitro of actinomycetes from nitrogen-fixing root nodules. Nature (London) 281:76-78.

5. Becking, J. H. 1970. Frankiaceae fam. nov. (Actinomycetales) with one new combination and six new species of the genus Frankia Brunchorst 1886, 174. Int. J. Syst. Bacteriol. 20:201-220.

6. Berry, A., and J. G. Torrey. 1979. Isolation and characterization in vivo and in vitro of an actinomycetous endophyte from Alnus rubra Bong, p. 69-83. In J. C. Gordon, C. T. Wheeler, and D. A. Perry (ed.), Symbiotic nitrogen fixation in the management of temperate forests. Forestry Research Laboratory, Oregon State University, Corvallis.

7. Callaham, D., P. Del Tredici, and J. G. Torrey. 1978. Isolation and cultivation in vitro of the actinomycete causing root nodulation in Comptonia. Science 199: 899-902.

8. Goldman, M. 1968. Fluorescent antibody methods. Academic Press, Inc., New York.

9. Lalonde, M., R. Knowles, and J.-A. Fortin. 1975. Demonstration of the isolation of non-infective Alnus crispa var. mollis Fern. nodule endophyte by morphological immuno-labelling and whole cell composition studies. Can. J. Microbiol. 21:1901-1920.

10. Lechevalier, M. P., and H. A. Lechevalier. 1979. The taxonomic position of the actinomycetic endophytes, $p$. 111-122. In J. C. Gordon, C. T. Wheeler, and D. A. Perry (ed.), Symbiotic nitrogen fixation in the management of temperate forests. Forestry Research Laboratory, Oregon State University, Corvallis.

11. Munoz, J. 1971. Double diffusion in plates. Methods Immunol. Immunochem. 3:146-160. 